\title{
The Acoustic Technology for Ctcs Isolation in Blood: Low-Cost Devices
}

\section{ISSN: 2637-773X}

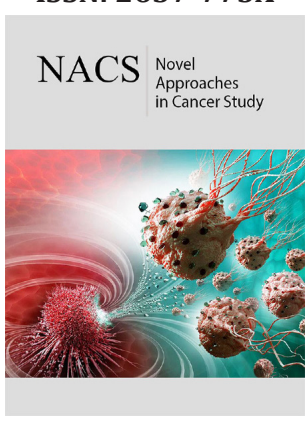

*Corresponding author: Itziar González, Institute of Physical and Information Technologies (ITEFI), Madrid, Spain

Submission: 监 June 12, 2019

Published: 㘹June 19, 2019

Volume 3 - Issue 1

How to cite this article: Itziar G, Alberto $P$, Sergio C, Julie E. The Acoustic Technology for Ctcs Isolation in Blood: Low-Cost Devices. Nov Appro in Can Study. 3(1). NACS.000551.2019.

DOI: 10.31031/NACS.2019.03.000551

Copyright@ Itziar González, This article is distributed under the terms of the Creative Commons Attribution 4.0 International License, which permits unrestricted use and redistribution provided that the original author and source are credited.

\section{Itziar González ${ }^{1 *}$, Alberto Pinto ${ }^{1}$, Sergio Cuellar ${ }^{1}$ and Julie Earl ${ }^{2,3}$}

${ }^{1}$ Institute of Physical and Information Technologies (ITEFI), Spain

${ }^{2}$ Molecular Epidemiology and Predictive tumor markers group, Ramón y Cajal Health Research Institute (IRYCIS), Madrid, Spain

${ }^{3}$ Biomedical Research Network in Cancer (CIBERONC), Madrid, Spain

\section{Abstract}

Blood samples can be used as a liquid biopsy in cancer diagnosis and chemotherapy monitoring. This label-free method offers benefits over traditional tissue invasive biopsy. It is possible to separate rare cells from blood samples by Ultrasounds on the basis of their physical properties in a biocompatible manner. A successful separation of cultured cancer cells from WBCs with acoustic-based methods is being demonstrated during the last years through different technological approaches. The concept of plate acoustic waves (PAW) applied to acoustophoresis was recently introduced to perform acoustic flow-through separation of rare cells in blood samples. It lies in the geometrical chip design, different to other micro separators (BAW and SAW). This new strategy allows soft materials of extremely reduced volume and low-cost fabrication and opens a door to printing manufacturing processes.

\section{Mini Review}

The use of blood samples as liquid biopsy for cancer diagnosis offers benefits over traditional tissue biopsy. Tumor cells are shed from primary distant sites in the bloodstream to circulate, becoming biomarkers of interest for cancer prognostics, monitoring treatment response in personalized medicine and contain information about possible specific mutations of a tumor Thus, isolation of viable circulating tumor cells (CTCs) is essential for liquid biopsy, which has shown to be an efficient alternative to tissue biopsy [1]. Chemotherapy and other therapy treatments, as well as mechanisms of treatment resistance can be monitored through the analysis of CTCs. However, isolation of CTCs from blood is nontrivial due to their extreme rarity in comparison to the blood cells [2].

Several attempts have been developed during the last decade to address it. Numerous microfluidic platforms are being developed during the last decade for isolating and capturing CTCs However, new devices for high-throughput CTC detection and isolation is still demanded. These systems must meet five goals to isolate viable CTC of a high-purity for clinical applications: the highest efficiency, purity, sensitivity, cell viability and throughputspeed. In addition, new low-cost approaches are required for translational clinic applications of diagnostic and monitoring. High purity is in strong demand for CTC enumeration, molecular characterization, and functional assays with less background intervention from WBCs.

Microfluidic devices provide numerous advantages over conventional methods associated to a reduction of the device size and simplification of protocols commonly used to perform cell sorting. These systems can be roughly classified into two groups, one of them comprises affinity-based methods that use surface markers, such as EpCAM, to distinguish CTCs from their surrounding blood cells. Studies performed on cells forming a tumor have demonstrated that epithelial tumor cells exhibit epithelial properties and express on their surface molecules of epithelial origin $[3,4]$. While these methods show very good specificity, they are hindered from false-negatives due to downregulation of the expression of surface markers on some CTCs which are undergoing epithelial-mesenchymal-transition (EMT) [3].

Many other strategies for detecting CTC in peripheral blood use differential physical cell properties in order to distinguish CTC from blood cells, including the cell size (epithelial cells) [4] their shape, deformability and/or density differences of CTCs and white blood cells (WBCs) and electrophoresis platforms [5]. These size-based are label-free methods, which 
include hydrodynamic and cross-flow filtration [6,7] using spiral channelization [8-13] channels that incorporate contraction/ expansion reservoirs for pinch alignment of the cells, micropillars [14-16], micro-scale vortices [17,18], serpentine microfluidic channels [19,20] or membrane microfilters [21] with pore diameters chosen to have the dimension between the diameters of cancer cells and blood cells. Some of them base their work on a deterministic lateral displacement (DLD) [22,23]. inertial focusing systems [24-26] or micro post trapping [27].

The acoustic sorting method offers a means to separate cells on the basis of their physical properties in a label-free, contactless, and biocompatible manner. A successful separation of cultured cancer cells from WBCs with acoustic-based methods was recently demonstrated in some microfluidic platforms using Bulk acoustic waves (BAW) and Surface acoustic waves (SAW) [28-30], applied either on cultured cancer cells or tumor cells in spiked blood samples respectively. These methods base their actuation on processes of differentiated cell enrichment induced by ultrasounds on flowing samples, involving mass transfer processes between parallel flows in some cases. The acoustic force is used to drive and collect target cells operating on size, density and compressibility of different cell populations. In a recent work we have successfully demonstrated the separation of CTCs from blood samples based on cell size [31] using Plate acoustic waves (PAW) of the thin structure of the chip. In our work, we reported a three-flow microfluidic (3FM) system established in a low-cost polymeric chip for the separation of CTCs with high purity based on the application of ultrasounds. The acoustic wave was strategically applied inside the channel along its central axis, where the TCs collected, leaving the blood cells circulating beside the channel walls to be separately extracted through different outlets. The microchannel takes advantage of a forced migration of cells [32] induced by the acoustic pressure gradient established. We evaluated the sensitivity and efficiency of CTC capture in a model system using blood samples from healthy donors spiked with tumor cell lines. 20 model system samples were tested for determining the recovery rate of the microdevice.

\section{Conclusion}

The acoustic technology for sorting purposes works at power intensities and frequencies similar to the ultrasonic imaging, with a little impact on the viability (high biocompatibility) $[31,32]$ of the cells. It presents clinical advantages as does not require modification of the media, thus no labeling, maximizing the potential of CTCs to be maintained in their native states, cultured, and analyzed in vitro or ex vivo. This non-contact and label-free separation of tumor cells from blood enables their recovery regardless of their molecular profile. It offers the potential of early detection of cancer and micrometastasis as well as noninvasive monitoring of the cancer patients undergoing treatment at a low-cost of fabrication, even allowing printing processing.

\section{Acknowledgement}

This work was partially supported by the Ministry of Economy and Competence of Spain (MINECO-MICINN) under Projects DPI2017-90147-R: “Low Intensity Ultrasounds for early diagnosis and control of Tumor and Stroma", a Research Collaboration established between the CSIC-RESULT Group and the Medical Oncology Department of the Hospital Universitario Ramón y Cajal of Madrid. The authors have no conflicts of interest.

\section{References}

1. Tang CM, Zhu P, Li S, Makarova OV, Amstutz PT, et al. (2018) Blood-based biopsies-clinical utility beyond circulating tumor cells. Cytometry A 93(12): 1246-1250.

2. Den Toonder J (2011) Circulating tumor cells: The grand challenge. Lab Chip 11(3): 375- 377.

3. Poudineh M, Sargent EH, Pantel K, Kelley SO (2018) Profiling circulating tumour cells and other biomarkers of invasive cancers. Nat Biomed Eng 2(2): $72-84$.

4. Lin HK, Zheng S, Williams AJ, Balic M, Groshen S, et al. (2010) Portable filter-based microdevice for detection and characterization of circulating tumor cells. Clin Cancer Res 16(20): 5011-5018.

5. Alshareef M, Metrakos N, Juarez Perez E, Azer F, Yang F, et al. (2013) Separation of tumor cells with dielectrophoresis-based microfluidic chip. Biomicrofluidics 7(1): 11803.

6. Li X, Chen W, Liu G, Lu W, Fu J (2014) Continuous-flow microfluidic blood cell sorting for unprocessed whole blood using surface-micromachined microfiltration membranes. Lab Chip 14: 2565-2575.

7. Tang CM, Zhu P, Li S, Makarova OV, Amstutz PT, et al. (2017) Filtration and analysis of circulating cancer associated cells from the blood of cancer patients. In: Prickril B, Rasooly A (Eds.), Biosensors and biodetection: Methods and protocols, electrochemical, bioelectronic, piezoelectric, cellular and molecular biosensors. $\left(2^{\text {nd }}\right.$ edn), Humana Press, New York, USA, 2: 511-524.

8. Chen H, Baoshan C, Sun B, Yapeng C, Ke Yang, et al. (2017) Highly sensitive capture of circulating tumor cells using micro-ellipse filters. Sci Rep 7: 610.

9. Warkiani ME, Guan G, Luan KB, Lee WC, Bhagat AA, et al. (2014) Slanted spiral microfluidics for the ultra-fast, label-free isolation of circulating tumor cells. Lab Chip 14(1): 128-137.

10. Gossett WM, Weaver AJ, Mach SC, Hur HT, Kwong Tse, et al. (2010) Labelfree cell separation and sorting in microfluidic systems. Anal Bioanal Chem 397(8): 3249-3267.

11. Hou HW, Warkiani ME, Khoo BL, Li ZR, Soo RA, et al. (2013) Isolation and retrieval of circulating tumor cells using centrifugal forces. Sci Rep $3: 1259$

12. Warkiani ME, Guan G, Luan KB, Lee WC, Bhagat AA, et al. (2014) Slanted spiral microfluidics for the ultra-fast, label-free isolation of circulating tumor cells. Lab Chip14(1): 128-137.

13. Khoo BL, Warkiani ME, Tan DS, Bhagat AA, Irwin D, et al. (2014) Clinical validation of an ultra-high-throughput spiral microfluidics for the detection and enrichment of viable circulating tumor cells. PLoS One9(7): e99409.

14. Bhagat AS, Hou HW, Li LD, Lim CT, Han J (2011) Pinched flow coupled shear-modulated inertial microfluidics for high-throughput rare blood cell separation. Lab Chip 11(11): 1870-1878.

15. Sarioglu AF, Aceto N, Kojic N, Donaldson MC, Zeinali M, et al. (2015) A microfluidic device for label-free, physical capture of circulating tumor cell clusters. Nature Methods12(7): 685-691.

16. Kim B, Lee JK, Choi S (2016) Continuous sorting and washing of cancer cells from blood cells by hydrophoresis. Bio Chip Journal 10(2): 81-87.

17. Stott SL, Hsu CH, Tsukrov DI, Yu M, Miyamoto DT, et al. (2010) Isolation of circulating tumor cells using a microvortex-generating herringbonechip. Proc Natl Acad Sci USA 107(43): 18392-18397. 
18. James C, Victor Yu, Manjima D, Corinne R, Melissa M, et al. (2016) Classification of large circulating tumor cells isolated with ultra-high throughput microfluidic vortex technology. Oncotarget 7(11): 1274812760.

19. Jason S, Yongxi Z, Perry GS, Laiying Ng, David SW, et al. (2010) Deformability considerations in filtration of biological cells. Lab on a Chip 10(7): 837-842.

20. Dessitter I, Guerrouahena BS, Benali N, Weschler J, Janne PA Y, et al. (2011) A New device for rapid isolation by size and characterization of rare circulating tumor cells. Anticancer Research 31(2): 427-442.

21. Zheng S, Lin H, Liu JQ Balic M, Datar R, etal. (2007) Membrane microfilter device for selective capture, electrolysis and genomic analysis of human circulating tumor cells. J Chromatogr A 1162 (2): 154-161.

22. Liu Z, Huang F, Du J, Shu W, Feng H, et al. (2013) Rapid isolation of cancer cells using microfluidic deterministic lateral displacement structure. Biomicrofluidics 7(1): 11801.

23. Okano H, Konishi T, Suzuki T, Ariyasu S, Aoki S, et al. (2015) Enrichment of circulating tumor cells in tumor-bearing mouse blood by a deterministic lateral displacement microfluidic device. Biomed Microdevices 17(3): 9964.

24. Zhang J, Sheng Y, Dan Y, Gursel A Nam Trung N, et al. (2016) Fundamentals and applications of inertial microfluidics: a review. Lab Chip 16(1): 1034.
25. Zhou J, Papautsky I (2013) Fundamentals of inertial focusing in microchannels. Lab Chip 13(6): 1121-1132.

26. Tu C, Zhou J, Liang Y, Huang B, Fang Y, et al. (2017) A flexible cell concentrator using inertial focusing. Biomed Microdevices 19(4): 83.

27. Tang Y, Jian S, Sisi L, Li W, Yvon EC, et al (2014) Microfluidic device with integrated microfilter of conical-shaped holes for high efficiency and high purity capture of circulating tumor cells. Sci Rep 4: 6052.

28. González I, Fernández LJ, López N, Berganzo J, Martin A, et al. (2011) Ultrasonic extraction of circulating tumor cells fom peripheral blooda noninvasive method to keep the cells viable for later biomolecular analysis. Proc $6^{\text {th }}$ Int Conf Microtech in Med and Biol MMB 83: 60-61.

29. Ding X, Peng Z, Lin SCS, Geri M, Li S, et al. (2015) Cell separation using tilted angle standing surface acoustic waves. Proc Natl Acad Sci USA 111(36): 12992-12997.

30. Li P, Mao Z, Peng Z, Zhou L, Chen Y, et al. (2015) Acoustic separation of circulating tumor cells. Proc Natl Acad Sci USA 112(16): 4970-4975.

31. Gonzalez I, Earl J, Pinto A, Fernandez LJ, Sainz B, et al. (2018) A disposable thin chip for rapid isolation of tumor cells and aggregates by ultrasounds. Micromachines (Basel) 9(3): 129.

32. Hulström J, Manneberg O, Dopf K, Hertz H, Brismar H, et al. (2007) Ultrasound in Med Biol 33(1): 145-151. 